\title{
Signals and prepatterns: new insights into organ polarity in plants
}

\author{
Aman Y. Husbands, ${ }^{1}$ Daniel H. Chitwood, ${ }^{1,2}$ Yevgeniy Plavskin, ${ }^{1,2}$ and Marja C.P. Timmermans ${ }^{1,2,3}$ \\ ${ }^{1}$ Cold Spring Harbor Laboratory, Cold Spring Harbor, New York 11724, USA; ${ }^{2}$ Watson School of Biological Sciences, Cold Spring \\ Harbor, New York 11724, USA
}

The flattening of leaves results from the interaction between upper (adaxial) and lower (abaxial) domains in the developing primordium. These domains are specified by conserved, overlapping genetic pathways involving several distinct transcription factor families and small regulatory RNAs. Polarity determinants employ a series of antagonistic interactions to produce mutually exclusive cell fates whose positioning is likely refined by signaling across the adaxial-abaxial boundary. Signaling candidates include a mobile small RNA-the first positional signal described in adaxial-abaxial polarity. Possible mechanisms to polarize the incipient primordium are discussed, including meristem-derived signaling and a model in which a polarized organogenic zone prepatterns the adaxial-abaxial axis.

The flattening of the leaf has been an important innovation in the evolution of land plants. The extension of the lamina is an outcome of dorsoventral (adaxial-abaxial) patterning of the developing primordium. Establishment of adaxial-abaxial polarity also directs the differentiation of distinct cell types within the leaf's adaxial/top and abaxial/bottom domains (Waites and Hudson 1995). For example, water-conducting xylem tissue in the vasculature forms adaxially to the sugar-bearing phloem cells. In many plant species, the adaxial side of the leaf also develops a thickened waxy cuticle and a tightly packed layer of palisade mesophyll cells that optimizes the capture of light, while the abaxial side contains loosely packed spongy mesophyll and a higher density of stomatal pores to facilitate gas exchange and regulate transpiration (Fig. 1A,B). The differentiation of these distinct cell fates and the extension of the leaf lamina are important adaptations that maximize photosynthesis while minimizing water loss to the environment.

Unlike animals, plants exhibit indeterminate growth and continuously give rise to new organs, such as leaves, from their shoot. The growing tip of the plant shoot, the shoot apical meristem (SAM), contains a population of

[Keywords: Organ polarity; pattern formation; signaling network; small RNAs]

${ }^{3}$ Corresponding author.

E-MAIL timmerma@cshl.edu; FAX (516) 367-8369.

Article is online at http://www.genesdev.org/cgi/doi/10.1101/gad.1819909. pluripotent stem cells that divide to replenish themselves and to provide a persistent source of daughter cells for the formation of new organ primordia (see Maughan et al. 2006). Lateral organs arise at the meristem periphery and become patterned along the adaxial-abaxial axis early in development while the primordium is still closely associated with the meristem. As primordia arise on the flank of the SAM, their adaxial side develops in closer proximity to the meristem tip than their abaxial side, leading botanists as early as the 1940s to suggest that this inherent asymmetry may direct the patterning of the leaf (Fig. 1C,D; Wardlaw 1949).

The first insights into the mechanisms that establish adaxial-abaxial polarity came from elegant surgical experiments conducted soon after. Incipient leaves separated from the meristem by a small incision emerge as abaxialized organs, which suggested the idea that a meristem-derived positional signal, now referred to as the Sussex signal, is required to specify adaxial fate (Sussex 1951, 1954). In addition, such surgically isolated primordia display radial symmetry, implying that abaxial cell fate alone is not sufficient to direct laminar outgrowth in the developing leaf. A recent extension of the Sussex experiments specifically implicates the epidermal layer (L1) in adaxial fate specification, as ablation of L1 cells between incipient primordia and the meristem similarly results in the formation of centric, abaxialized organs (Reinhardt et al. 2005). Interestingly, proximal regions of primordia separated at the P1 stage of development remain abaxialized, despite the correct adaxial-abaxial polarization of the leaf's distal end (" $\mathrm{P}$ " referring to plastochron stage or the time between subsequent leaf initiations, such that $\mathrm{PO} / \mathrm{I} 1$ is the incipient primordium; $\mathrm{P} 1$, the first primordium visibly protruding from the SAM; P2, the second oldest primordium; etc.). This suggests that the acquisition of organ polarity occurs gradually and does not spread from distal to proximal regions of primordia, but rather depends on sustained positional information provided by the meristem. Such positional information is, however, required over only a short developmental window. Surgical experiments indicate that by the $\mathrm{P} 2$ stage of primordium development, mechanisms within the organ are in place to maintain the separation of adaxial and abaxial domains throughout organ development (Reinhardt et al. 2005). 

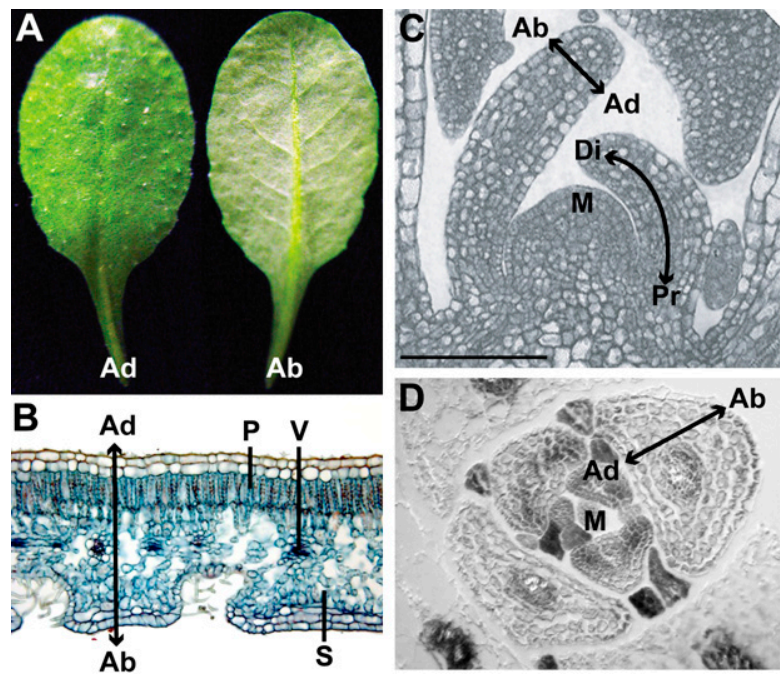

Figure 1. Adaxial-abaxial leaf polarity. $(A)$ The adaxial side of an Arabidopsis leaf is dark green and trichome-rich, whereas the abaxial leaf surface is gray-green and trichome-poor. $(B)$ Transverse section through a Nerium leaf illustrating the differentiation of distinct cell types within the adaxial and abaxial domains. Rectangular palisade mesophyll $(\mathrm{P})$ cells form a tightly packed file beneath the adaxial epidermis, whereas spongy mesophyll (S) cells separated by large intercellular air spaces differentiate abaxially. Within the vasculature $(\mathrm{V})$, water-bearing xylem cells differentiate adaxial to sugar-bearing phloem cells. (C) Longitudinal section of an Arabidopsis apex, showing the proximal-distal and adaxial-abaxial axes of leaf primordia relative to the SAM (marked $M$ ). (D) Transverse section of an Arabidopsis vegetative shoot apex, showing the positions of the adaxial and abaxial sides of leaf primordia relative to the meristem (M). Note the spiral phyllotaxis of leaves around the SAM with increasingly older primordia at a greater distance from the meristem. (The image in $B$ is used with permission from the University of Wisconson Plant Teaching Collection at http://botit.botany.wisc.edu/images/130/Leaf.)

While the nature of the positional information from the meristem that polarizes the developing primordium remains unknown, genes involved in adaxial-abaxial patterning have been identified in evolutionarily diverse model organisms, such as Antirrhinum majus (snapdragon), Arabidopsis thaliana, Zea mays (maize), and Oryza sativa (rice). Among these polarity determinants are several transcription factor and small RNA families that act in conserved and partially redundant genetic pathways to promote adaxial or abaxial fate. Here, we review the contributions of these genetic pathways to adaxial-abaxial patterning in diverse plant lineages, highlighting in particular the unique role of small regulatory RNAs. We propose that the maintenance of precisely defined adaxial and abaxial domains throughout primordium development is achieved through mutually antagonistic relationships between polarity determinants and signaling across the adaxial-abaxial boundary, perhaps mediated by a mobile small RNA. Finally, we present possible patterning mechanisms that set up the initial polarization of the incipient primordium, including patterning via a meristem-derived adaxializing signal and a model in which the organogenic zone of the SAM is prepatterned along the adaxial-abaxial axis.

\section{Transcriptional regulators of leaf polarity}

\section{Adaxial determinants}

PHANTASTICA. The first gene recognized to function in the control of leaf polarity-PHANTASTICA (PHAN), which encodes a MYB domain transcription factor-was cloned from Antirrhinum nearly 50 years after surgical experiments suggested the existence of a meristemderived adaxializing signal (Waites and Hudson 1995; Waites et al. 1998). phan mutants display a range of phenotypic severities, governed in part by temperature and leaf position. Basally positioned phan leaves exhibit a weak polarity phenotype, developing adventitious blade outgrowths on their upper surface. These outgrowths are associated with sectors of cells that have lost adaxial fate and instead have taken on abaxial identity. The most severe phan mutant leaves completely lack flattened lamina and show radial symmetry with abaxial cell types encircling central xylem tissue (see Fig. 2A,B for comparable phenotypes in maize). These phenotypes demonstrate that PHAN is necessary to specify adaxial fate in Antirrhinum, and support the idea that extension of the leaf blade results from the juxtaposition of adaxial and abaxial tissues (Waites and Hudson 1995). Exactly how an adaxial-abaxial boundary coordinates blade outgrowth, however, is still unclear. In addition to their polarity
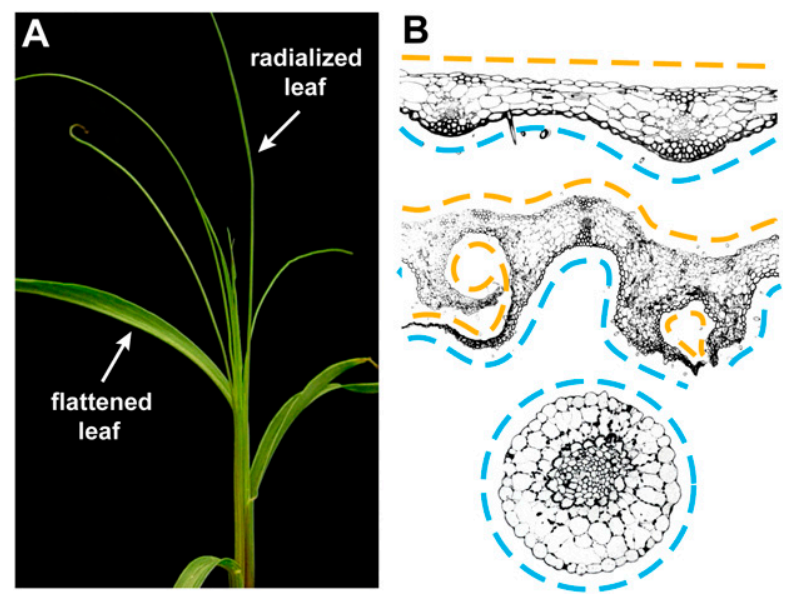

Figure 2. Phenotypes of mutants with perturbed adaxialabaxial patterning. (A) Normal maize leaves develop flattened blades due to interaction between the adaxial and abaxial domains. $1 b 11$ mutants interfere with ta-siRNA biogenesis and adaxial cell fate specification and consequently their leaves are often radial and abaxialized. (B) Transverse sections through a wild-type (top), weakly adaxialized mwp1 (middle), and fully abaxialized lbl1 (bottom) leaf. Note the formation of ectopic blade outgrowths at the boundaries of adaxialized tissue sectors on the abaxial leaf surface of the mwp1 leaf (marked by orange lines), and the radial symmetry of the $1 b 11$ leaf. (Orange lines) Adaxial; (blue lines) abaxial. (The images in $B$ are reproduced with permission from Candela et al. [2008] [ㄷ 2008 American Society of Plant Biologists] and Timmermans et al. [1998].) 
phenotypes, the most severe phan alleles frequently lead to meristem arrest. This observation, in conjunction with data from surgical experiments, points to a mutually promotive relationship between adaxial identity and meristem activity.

The role of PHAN in adaxial-abaxial patterning is conserved in tobacco, tomato, and several other compound-leafed species (Kim et al. 2003a; McHale and Koning 2004; Hay and Tsiantis 2006). Surprisingly, mutations in the maize and Arabidopsis PHAN orthologs ROUGH SHEATH2 (RS2) and ASYMMETRIC LEAVES1 (AS1), respectively, cause no obvious polarity defects (Timmermans et al. 1999; Tsiantis et al. 1999; Byrne et al. 2000). PHAN, RS2, and AS1 are expressed uniformly throughout developing primordia, suggesting that any contributions to adaxial-abaxial patterning are regulated by interacting protein partners. Supporting this, AS1 and RS2 interact with AS2, a LOB domain transcription factor that localizes to the adaxial-most cell layers of young leaf primordia (Xu et al. 2003; Phelps-Durr et al. 2005; Husbands et al. 2007; Iwakawa et al. 2007). Although organ polarity is not obviously perturbed in as 1 as 2 double mutants, Arabidopsis plants that constitutively express AS2 develop leaf and vascular defects consistent with an adaxialized phenotype (Lin et al. 2003). These findings indicate that the AS pathway in Arabidopsis contributes to organ polarity, but does so redundantly with other genetic pathways. Indeed, screens for mutations that enhance the as 1 or as 2 defects have implicated several regulatory networks, including chromatin-remodeling, RNAi, and protein synthesis pathways in the regulation of adaxial-abaxial cell fate (Fig. 3A; Li et al. 2005; Garcia et al. 2006; Ueno et al. 2007; Pinon et al. 2008). Some of these are discussed elsewhere in this review and illustrate how molecularly conserved polarity determinants can have diverse contributions to adaxial-abaxial patterning in divergent species.

The class III homeodomain-leucine zipper (HD-ZIPIII) genes. The prominent contribution of PHAN to adaxial fate in Antirrhinum is mirrored by that of members of the HD-ZIPIII family of transcription factors in Arabidopsis, rice, and maize (McConnell et al. 2001; Emery et al. 2003; Juarez et al. 2004a,b; Itoh et al. 2008). The HD-ZIPIII genes were recognized as polarity determinants based on the phenotypes resulting from semidominant gain-offunction mutations. Arabidopsis plants expressing such dominant alleles of PHABULOSA (PHB), PHAVOLUTA $(P H V)$, or REVOLUTA (REV) possess an enlarged SAM and develop adaxialized lateral organs, due to the altered expression of mutant transcripts (McConnell et al. 2001; Emery et al. 2003). PHB, PHV, and REV are normally expressed on the adaxial side of developing leaf primordia. However, transcripts derived from gain-of-function HDZIPIII alleles accumulate ectopically on the abaxial side as well, indicating that $P H B, P H V$, and $R E V$ are sufficient to specify adaxial cell fate (McConnell et al. 2001; Juarez et al. 2004a; Itoh et al. 2008).

HD-ZIPIII genes are expressed not only on the adaxial side of leaf primordia, but also within the tip of the meristem, and $P H B$ forms rays of expression that connect the meristem with predicted sites of organ initiation. This pattern of expression presents the possibility that the HD-ZIPIII genes coordinate the bidirectional communication between the SAM and the adaxial side of organ primordia (McConnell et al. 2001). As HD-ZIPIII
Figure 3. A network of conserved transcription factors and small RNA pathways maintains adaxialabaxial polarity. (A) The HD-ZIPIII, AS, and TAS3 tasiRNA pathways contribute to the specification of adaxial cell fate. In Arabidopsis, HD-ZIPIII activity is regulated via a negative feedback loop involving the ZPR proteins, while the PIGGYBACK ribosomal proteins (PGY) (Pinon et al. 2008), ASYMMETRIC LEAVES ENHANCER3 (AE3) (Huang et al. 2006), and histone deacetylase proteins (HDAC) (Ueno et al. 2007) enhance the AS pathway (dotted outline). Members of the KANADI and ARF families, together with the miRNA miR166, contribute to the specification of abaxial identity. The site of YABBY activity varies between species but its contribution to organ outgrowth may be conserved. Antagonistic interactions between the polarity determinants create mutually exclusive adaxial and abaxial cell fates that contribute to the stable maintenance of organ polarity throughout development. Direct interactions are marked with a bold line. $(B)$ Diagram of the TAS3 tasiRNA pathway. miR390-loaded AGO7 targets TAS3 transcripts, which upon cleavage of the $3^{\prime}$ target site are converted into dsRNAs through the activities of RDR6 and SGS3/LBL1 and subsequently processed by DCL4 into phased 21-nt species. The TAS3-derived ta-siRNAs, tasiR-ARFs, act in trans to repress the expression of the abaxial determinants ARF3 and ARF4.
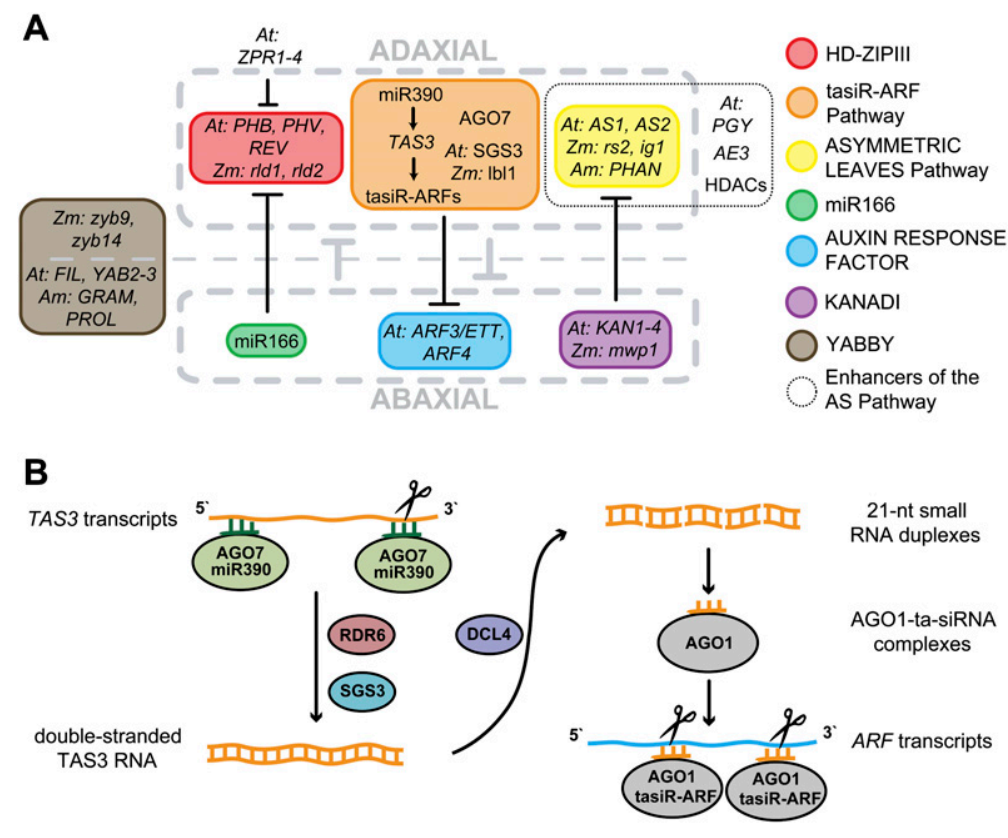
proteins contain a putative lipid/sterol-binding START domain (Ponting and Aravind 1999; Schrick et al. 2004), part of this communication could include the control of HD-ZIPIII activity via a mobile lipid signal. Perhaps reflective of their prominent role in adaxial fate specification, HD-ZIPIII activity is subject to additional levels of regulation. The nature of the dominant HD-ZIPIII mutations revealed microRNA (miRNA)-mediated regulation at the post-transcriptional level (see below), and more recent studies have shown that HD-ZIPIII function in Arabidopsis is further modulated by interaction with LITTLE ZIPPER (ZPR) proteins. These small leucine zipper-containing proteins prevent HD-ZIPIII dimerization, an obligate requirement for the binding of these polarity determinants to DNA (Wenkel et al. 2007; Kim et al. 2008). ZPR expression is induced by HD-ZIPIII activity, suggesting that a negative feedback loop modulates HD-ZIPIII function. Although the enlarged SAMs of zpr loss-of-function mutants illustrate the significance of this feedback loop in meristem regulation, its contribution to organ polarity is less clear.

The replacement of adaxial cell types with their abaxial counterparts in phan loss-of-function mutants and the converse effects seen in HD-ZIPIII gain-of-function mutants suggest that adaxial and abaxial cell fates are mutually exclusive. Negative feedback regulation, as observed between the HD-ZIPIII and ZPR genes, could conceivably separate adaxial and abaxial identities defining distinct domains of activity. However, ZPR1 and $Z P R 3$ transcripts accumulate late in primordium development and only adaxially (Wenkel et al. 2007; Kim et al. 2008), instead suggesting a role for these proteins in finetuning HD-ZIPIII function. Insights into the molecular basis underlying the mutual exclusivity of adaxial and abaxial cell fates came from analyses of determinants involved in the specification of abaxial identity.

\section{Abaxial determinants}

The KANADI (KAN) genes. The KAN genes are contributors to abaxial identity in both monocot and dicot plant species (Kerstetter et al. 2001; Eshed et al. 2001, 2004; Candela et al. 2008; Zhang et al. 2009). These genes encode transcription factors containing a MYB-like GARP DNA-binding domain and are expressed in the abaxial domain of lateral organs. Mutations in the KAN family members milkweed pod1 from maize and SHALLOT-LIKE1 from rice lead to the formation of partially adaxialized leaves (Fig. 2B; Candela et al. 2008; Zhang et al. 2009). A role for the four Arabidopsis KAN genes in adaxial-abaxial patterning is clear from phenotypes of higher-order mutants. Double and triple mutants develop ectopic outgrowths on their abaxial leaf surface or produce radially symmetric adaxialized organs. The latter phenotype closely resembles those of HD-ZIPIII gain-of-function mutants; indeed, HD-ZIPIII transcripts accumulate ectopically throughout radialized kan1 kan2 organs (Eshed et al. 2001, 2004). Conversely, ectopic expression of KAN1 or KAN2 throughout the developing leaf leads to fully abaxialized organs, with a concomitant loss of HD-ZIPIII expression, implicating the KAN genes as possible negative regulators of the HD-ZIPIII genes (Kerstetter et al. 2001; Eshed et al. 2004). Likewise, lossof-function $\mathrm{phb}$ phv rev triple-mutant embryos develop a single abaxialized cotyledon in lieu of a meristem-a phenotype that can be suppressed by additional mutations in kan1, kan2, and kan4 (Emery et al. 2003; Izhaki and Bowman 2007). These genetic interactions point to a reciprocal role for the HD-ZIPIII genes in excluding KAN gene expression from the SAM to maintain meristem activity. An antagonistic relationship between these polarity determinants in the developing leaf could provide a possible basis for the mutually exclusive and opposing nature of adaxial and abaxial cell fates.

A further antagonistic interaction has been reported recently between KAN proteins and the AS pathway. KAN1 binds a cis-element in the promoter of AS2 to repress its expression at the transcriptional level, thereby restricting AS2 activity to the adaxial side of leaves $(\mathrm{Wu}$ et al. 2008). This transcriptional repression of AS2 by KAN1 is likely to represent only a single facet of its role in leaf polarity, as as 2 mutants fail to suppress the kan1 kan2 phenotype. KAN1 may negatively regulate adaxial determinants beside $A S 2$ or, alternatively, polarity determinants like the KAN genes may have dual activities and be required for the induction of abaxial-promoting pathways as well. These findings, in conjunction with the likely repressive nature of the AS pathway (Guo et al. 2008), illustrate the complexity of interactions between polarity determinants that lead to mutual exclusivity of adaxial and abaxial cell fates (Fig. 3A). These opposing cell fates, in turn, are likely an integral part of the mechanism that maintains the separation between adaxial and abaxial domains throughout organ development.

The AUXIN RESPONSE FACTORS (ARFs). A strikingly similar phenotype to that of the kan1 kan2 mutants can be seen in Arabidopsis plants mutant for the $A R F$ family members ARF3/ETTIN (ARF3) and ARF4 (Pekker et al. 2005). ARF genes encode transcription factors that control downstream responses to the plant hormone auxin, which regulates numerous developmental processes (for review, see Guilfoyle and Hagen 2007). arf3 single mutants suppress the KAN1 overexpression phenotype, positioning ARF3 as a potential downstream target of KAN activity (Pekker et al. 2005). However, neither ARF3 nor ARF4 expression is altered in a kan1 kan2 background. Further, expression of $A R F 3$ from a ubiquitously expressed promoter does not induce the formation of abaxialized lateral organs, as is the case with ectopic KAN1 or KAN2 expression (Pekker et al. 2005). This discrepancy is not simply explained by the fact that ARF3 and ARF4 are targets of small RNA-mediated gene regulation (see below), as the ubiquitous expression of a small RNA-insensitive ARF3 allele also negligibly affects adaxial-abaxial patterning (Fahlgren et al. 2006; Hunter et al. 2006). Together, these data suggest that ARF3, ARF4, and the KAN proteins have overlapping roles as abaxial determinants, but differ in their interactions with other components of the adaxial-abaxial patterning network. 
YABBY genes: downstream components in adaxialabaxial polarity. While the relative contributions to organ polarity of the factors described above may vary between species, their functions as adaxial or abaxial determinants are evolutionarily conserved. This is not the case for the YABBY genes. In Arabidopsis, members of this transcription factor family-including FILAMENTOUS FLOWER (FIL), YAB2, and YAB3-promote abaxial identity (Sawa et al. 1999; Siegfried et al. 1999). Higher-order yabby loss-of-function mutants show weak adaxialized phenotypes, while constitutive expression of YABBY proteins abaxializes lateral organs and terminates the meristem. Consistent with this activity, YABBY genes are expressed on the abaxial side of lateral organs in Arabidopsis. Abaxial-specific YABBY expression is conserved in a number of dicot species, including Antirrhinum, tomato, and tobacco (Kim et al. 2003b; Eshed et al. 2004; Golz et al. 2004; Navarro et al. 2004). In rice, however, YABBY genes show a nonpolarized expression pattern and their ectopic expression has no effect on adaxial-abaxial patterning (Yamaguchi et al. 2004; Dai et al. 2007). Most strikingly, the maize YABBY homologs $z y b 9$ and $z y b 14$ are expressed on the adaxial side of developing lateral organs (Juarez et al. 2004b), indicating that, although YABBY genes in Arabidopsis promote abaxial fate, this function is not conserved throughout the angiosperms.

Several observations suggest that the YABBY genes act downstream from other polarity determinants to direct blade outgrowth at the adaxial-abaxial boundary. Expression of YABBY genes begins later in primordium development than that of the HD-ZIPIII and KAN genes (Heisler et al. 2005; Toriba et al. 2007), and the ectopic blade outgrowths seen in weak polarity mutants show strong YABBY gene expression irrespective of whether such outgrowths arise on the upper or lower leaf surface (Eshed et al. 2004; Juarez et al. 2004b). In fact, YABBY genes are required for their production, as kan1 kan2 fil yab3 quadruple mutants lack the ectopic blade outgrowths seen in kan1 kan2 mutants (Eshed et al. 2004). The ancestral function of YABBY genes may therefore be to drive blade outgrowth along planes dictated by an adaxial-abaxial boundary, and in this regard it is interesting to note that YABBY expression in potato and maize becomes localized to the presumptive site of this boundary during primordium development (Eshed et al. 2004; Juarez et al. 2004b).

Thus, while the function of polarity determinants in specifying either adaxial or abaxial fate is conserved, their input into the regulation of YABBY genes must have diverged during plant evolution. In addition to regulating YABBY gene expression, adaxial and abaxial determinants employ a series of negative interactions to define mutually opposing cell fates (Fig. 3A). Although the molecular basis for the majority of these interactions remains unknown, they likely form part of a larger regulatory network that preserves the separation of adaxial and abaxial domains during primordium growth. This network must also include positive interactions that reinforce the identity within each domain, as well as signaling between the domains to provide positional inputs that refine the boundary. As none of the transcription factors described above are known to be mobile, their activities are likely patterned in response to positional signals. At least one of these signals is now known to be a mobile small RNA whose contribution to organ polarity is discussed below.

\section{Novel small RNA-based patterning mechanisms in adaxial-abaxial polarity}

\section{Small RNAs as adaxial and abaxial determinants}

Because HD-ZIPIII genes are sufficient to specify adaxial fate, their polarized expression is vital to the proper patterning of leaves. Dominant alleles that lead to ectopic abaxial expression of HD-ZIPIII transcripts result from mutations in a target site recognized by the miRNA miR166 (McConnell et al. 2001; Juarez et al. 2004a; Mallory et al. 2004; Itoh et al. 2008). In maize and Arabidopsis, mature miR166 accumulates on the abaxial side of leaf primordia in a pattern complementary to that of its HD-ZIPIII targets (Juarez et al. 2004a; Kidner and Martienssen 2004). miR166 directs the cleavage of HDZIPIII transcripts (Tang et al. 2003), and the functional significance of this relationship is demonstrated not only by the adaxializing phenotypes caused by dominant HDZIPIII alleles, but also by the development of severely abaxialized organs upon ectopic expression of miR166 (Alvarez et al. 2006). The negative regulation of HD-ZIPIII genes by miR166 therefore reveals an additional component of the regulatory network maintaining distinct adaxial and abaxial fates.

Given the significant role for miR166 in organ polarity, mechanisms must exist to precisely define its spatiotemporal pattern of accumulation. Insight into one such regulatory mechanism came from analyses of the maize mutant leafbladeless1 (1bl1). Severe recessive alleles of $1 b 11$ lead to the formation of centric abaxialized leaves in which HD-ZIPIII expression is dramatically reduced (Fig. 2A,B; Timmermans et al. 1998; Juarez et al. 2004b). $1 b 11$ encodes the functional ortholog of SUPPRESSOR OF GENE SILENCING3 (SGS3), an essential component in the Arabidopsis trans-acting siRNA (ta-siRNA) biogenesis pathway (Nogueira et al. 2007). This specialized RNAi pathway has been reviewed elsewhere (Chapman and Carrington 2007); however, in brief, miRNA-guided cleavage triggers conversion of ta-siRNA precursor (TAS) transcripts into long dsRNAs via an RNA-DEPENDENT RNA POLYMERASE6 (RDR6)- and 1bl1/SGS3-dependent pathway and sets the register for phased, 21-nucleotide (nt) tasiRNA production by DICER-LIKE4 (DCL4) (Peragine et al. 2004; Vazquez et al. 2004; Allen et al. 2005). tasiRNAs are then loaded into an ARGONAUTE (AGO)containing RNA-induced silencing complex (RISC) and, like miRNAs, act in trans to post-transcriptionally repress the expression of target genes (Fig. 3B).

The molecular identity and loss-of-function phenotype of $1 b 11$ demonstrate that ta-siRNAs act as adaxial determinants in maize. Their effect is mediated via miR166, as 
mir166c and mir166i precursors are specifically up-regulated in $1 b 11$ mutant apices and mature miR166 accumulates ectopically on the adaxial side of incipient and developing $1 b 11$ leaf primordia (Nogueira et al. 2007). Adaxial-abaxial polarity in the maize leaf thus employs a novel patterning mechanism, relying on the opposing activities of two distinct small RNAs: (1) ta-siRNAs that define the adaxial side of leaf primordia by spatially restricting the domain of miR166 accumulation; and (2) miR166, which in turn delineates the abaxial side by restricting expression of the adaxializing HD-ZIPIII genes.

Unlike maize, the contribution of ta-siRNAs to adaxialabaxial patterning in Arabidopsis is not immediately apparent. Mutations in ta-siRNA biogenesis components, such as $S G S 3$, confer subtle leaf development phenotypes consistent with an accelerated transition from the juvenile to the adult phase, but adaxial-abaxial patterning is not conspicuously altered (Peragine et al. 2004; Vazquez et al. 2004). The functional contributions of ta-siRNAs to leaf development are mediated through the subspecialized TAS3 ta-siRNA pathway, which requires the unique association of miR390 with its effector AGO7 to trigger ta-siRNA biogenesis (Figs. 3B, 4A; Allen et al. 2005; Adenot et al. 2006; Montgomery et al. 2008). A subset of TAS3 ta-siRNAs, termed tasiR-ARFs, targets the abaxial determinants ARF3 and ARF4 (Allen et al. 2005). Expression of $A R F 3$ transgenes insensitive to tasiR-ARF regulation results in vegetative phase change defects similar to ta-siRNA biogenesis mutants but does not disrupt adaxial-abaxial polarity (Fahlgren et al. 2006; Hunter et al. 2006). Exactly why the loss of tasiR-ARF activity in Arabidopsis confers no obvious leaf polarity phenotypes has yet to be resolved. Functional overlap exists between the TAS3 ta-siRNA and AS pathways, as tasiR-ARF biogenesis mutants enhance the as 1 and as 2 phenotypes and the expression levels of FIL are elevated specifically in mutants compromised for both pathways (Li et al. 2005; Garcia et al. 2006; Xu et al. 2006). Further, additively with the AS pathway, tasiR-ARFs in Arabidopsis repress levels of miR166, a function reminiscent of the contribution of $1 b 11$ to adaxial-abaxial patterning in maize $(\mathrm{Xu}$ et al. 2006). However, as the leaves of such double mutants largely retain correct adaxial-abaxial polarity, additional redundancies or differences in the patterning of the TAS3 ta-siRNA pathway and downstream targets must exist between Arabidopsis and maize.

\section{Small RNAs as generators of pattern}

In maize, adaxial-abaxial polarity in the incipient leaf is specified through a novel patterning mechanism in which miR390 is positioned at the top of a cascade of small RNA activities. miR390 localizes to the adaxial side of the incipient primordium, where it triggers the biogenesis of TAS3 ta-siRNAs that then restrict miR166 abaxially (Fig. 4C; Nogueira et al. 2007, 2009). As other components of the TAS3 ta-siRNA biogenesis pathway are expressed more broadly throughout the SAM, miR390 is the restrictive factor that precisely positions ta-siRNA accumulation. Surprisingly, miR390 remains polarized to

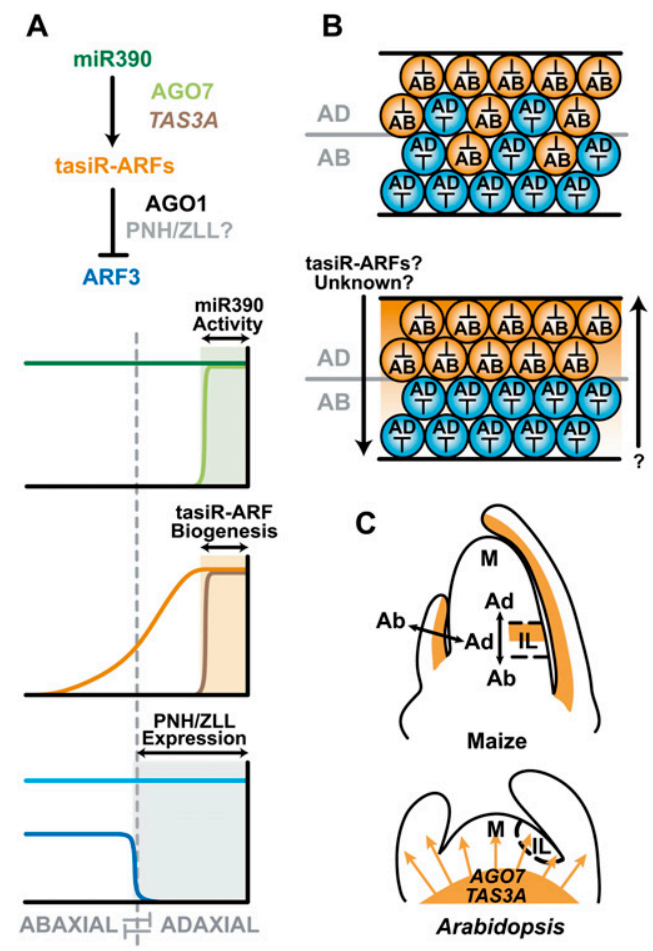

Figure 4. Diverse contributions of TAS3 ta-siRNAs to adaxialabaxial patterning. (A, top graph) In Arabidopsis, mature miR390 (dark green) accumulates throughout the leaf, but its activity (gray-green box) is restricted to the adaxial side by localized expression of AGO7 (light green). (Middle graph) tasiRARF biogenesis (pale orange box) is further confined to the two most adaxial cell layers by the restricted expression of the TAS3A precursor (brown). Mobility of tasiR-ARFs (orange) creates a gradient of accumulation across the developing leaf that is strongest near its adaxial site of biogenesis. (Bottom graph) This gradient yields regions of high and low tasiR-ARF activity, perhaps patterned in part via adaxial expression of PHN/ZLL (gray box), that restrict ARF3 protein accumulation (dark blue) to the abaxial side even though ARF3 transcripts (light blue) are present throughout leaf primordia. (B) The mutually opposing nature of adaxial and abaxial cell fates, resulting in part from antagonistic interactions between cellautonomous polarity determinants, contributes to the maintenance of organ polarity, but is unlikely sufficient to define a precise boundary between adaxial and abaxial organ domains. Superimposed intercellular signals that act between the domains can provide positional inputs to refine the adaxialabaxial boundary. The adaxially derived mobile tasiR-ARFs are candidates for such a signal and may act in conjunction with positional signals from the abaxial domain. $(C)$ In maize, miR390 and tasiR-ARFs (orange) accumulate adaxially in initiating and developing leaf primordia. In Arabidopsis, tasiR-ARFs move from their site of biogenesis below the SAM into the meristem proper and therefore accumulate uniformly throughout incipient primordia (IL).

the upper "adaxial" side of abaxialized $l b l 1$ primordia, suggesting that the polar accumulation of miR390 is regulated independently of the ta-siRNA pathway and downstream polarity determinants, such as miR166 and the HD-ZIPIII genes (Nogueira et al. 2009). miR390 
accumulation may instead be regulated by positional information inherent to the SAM. This positional information may act to pattern small RNA-related factors. Expression of the miR390 precursors in the SAM is not limited to the incipient primordium, indicating that the discrete accumulation of mature miR390 in just the incipient leaf is regulated at the level of small RNA processing and/or stability (Nogueira et al. 2009). Irrespective of the underlying mechanism, as ta-siRNAs delineate the adaxial and abaxial sides through repression of miR166, the precise spatiotemporal regulation of miR390 accumulation in the incipient primordium is critical to adaxial-abaxial patterning in maize.

While tasiR-ARFs in Arabidopsis do not overtly contribute to organ polarity, localization of TAS3 ta-siRNA pathway components reveals additional RNAi-based mechanisms underlying adaxial-abaxial patterning (Chitwood et al. 2009). Unlike maize, miR390 accumulates throughout the SAM and developing leaf primordia in Arabidopsis (Fig. 4C). The extent of miR390 activity is, however, curtailed by the highly restricted localization of AGO7 to the adaxial side of leaves and in the vasculature and pith region beneath the SAM. Likewise, TAS3A, the functional contributor of tasiR-ARFs during leaf development (Adenot et al. 2006; Addo-Quaye et al. 2008), localizes to the few adaxial-most cell layers of leaf primordia in a pattern similar to AGO7 (Chitwood et al. 2009). Thus, in contrast to maize, where the post-transcriptional regulation of miR390 accumulation polarizes the TAS3 ta-siRNA pathway, in Arabidopsis the localized expression of the miR390 substrate (TAS3A) and effector complex (AGO7) adaxially positions tasiR-ARF biogenesis.

An ARF3-based sensor for tasiR-ARF activity shows that these small RNAs act across the entire adaxialabaxial axis, but more so on the adaxial side of the leaf (Chitwood et al. 2009). As tasiR-ARF biogenesis is limited to the two adaxial-most cell layers of leaves, tasiR-ARF activity outside this defined region results from intercellular mobility of this small RNA. Indeed, tasiR-ARFs accumulate in a graded pattern across the leaf blade, strongest near their adaxial source of biogenesis and dissipating toward the abaxial side of the leaf. Additionally, tasiR-ARFs were found to act in the SAM, despite the absence of $A G O 7$ and TAS3A expression in the meristem, suggesting that tasiR-ARFs move from below the SAM into the meristem proper (Chitwood et al. 2009). Such movement might provide an additional explanation for the differing contributions of tasiR-ARFs to leaf polarity in Arabidopsis versus maize. Whereas tasiR-ARF activity in maize is polarized in incipient primordia, tasiR-ARFs in Arabidopsis are found uniformly throughout the SAM, and their contribution to adaxial-abaxial patterning may not be realized until later in organ development (Fig. 4C). The intercellular trafficking of tasiR-ARFs defines these small RNAs as the first mobile signal in adaxial-abaxial polarity. In addition to the mutual exclusivity of adaxial and abaxial cell fates, signaling between the adaxial and abaxial domains is likely required to maintain leaf polarity. tasiR-ARFs, possibly in conjunction with an abaxial-derived signal, may mediate such interdomain communication and sharpen the adaxial-abaxial boundary that drives laminar outgrowth (Fig. 4B).

\section{Interpreting the tasiR-ARF gradient}

Consistent with the idea that tasiR-ARF mobility refines the adaxial-abaxial boundary, the gradient of tasiR-ARF accumulation does not simply translate into an inverse expression gradient of its targets. Instead, it is interpreted into discrete regions of high and low tasiR-ARF activity that creates a sharply defined domain of ARF3 and ARF4 expression on the abaxial side of leaf primordia (Pekker et al. 2005; Chitwood et al. 2009). How tasiR-ARF activity becomes patterned in Arabidopsis leaves is not currently understood. This may involve regulation by small RNA effector complexes, just as miR390 activity is limited to the adaxial sides of leaves by the localized expression of AGO7 (Chitwood et al. 2009). tasiR-ARFs act through the ubiquitously expressed AGO1 but perhaps may also act via PINHEAD/ZWILLE/AGO10 (PNH/ZLL), which localizes specifically to the adaxial side of leaf primordia (Fig. 4A; Lynn et al. 1999; Montgomery et al. 2008). PNH/ZLL is required to repress $\mathrm{miR} 166$ accumulation in the meristem, a function reminiscent of that of $1 b l 1$ in maize (Nogueira et al. 2007; Liu et al. 2009). An additional link between PNH/ZLL and the tasiR-ARF pathway is suggested by the observation that ARF3 proteins, rather than ARF3 transcripts, are polarized by tasiR-ARFs (Pekker et al. 2005). In addition to AGO1, PNH/ZLL can mediate small RNA-guided translational repression (Brodersen et al. 2008). This finding, and the superposition of the tasiR-ARF gradient onto the localization of $\mathrm{PNH} / \mathrm{ZLL}$ and AGO1, could easily account for the discrete patterning of tasiR-ARF activity in leaves (Fig. 4A).

A more tantalizing concept to explain the conversion of the tasiR-ARF gradient into discrete regions of activity would be a morphogen-like patterning mechanism, more commonly found in animal systems. Mathematical modeling of the interaction between tasiR-ARFs and ARF3 supports the theoretical feasibility of such a scenario (Levine et al. 2007). With this in mind, it is interesting to note that ARF3 and ARF4 each possess two tasiR-ARF target sites (Allen et al. 2005). If tasiR-ARFs were to act in a combinatorial fashion on ARF3 and ARF4, this would further facilitate their concentration-dependent, morphogen-like patterning. Critical to such a concept is a dosedependent response in target expression to tasiR-ARF levels. Although the effect of tasiR-ARF dosage on leaf development is not known, target levels may be important, as increasing the levels of nontargeted ARF3 transcripts results in increasingly severe leaf defects (Fahlgren et al. 2006; Hunter et al. 2006). Should tasiR-ARFs prove to regulate their target levels in a concentration-dependent morphogen-like manner, this would also provide an efficient, flexible mechanism to position the boundary between the adaxial and abaxial domains of developing leaves (Fig. 4B).

Small RNAs play a prominent role in adaxial-abaxial patterning and, like their protein counterparts, are regulated at the level of biogenesis, stability, activity, and, in 
the case of tasiR-ARFs, mobility. tasiR-ARFs in Arabidopsis represent the first identified signaling component in the network of interactions that maintains the separation of the adaxial and abaxial domains throughout leaf development. Similar signals from the abaxial side may also be required to further refine the adaxial-abaxial boundary (Fig. 4B). In maize, ta-siRNAs are part of a small RNA cascade that polarizes miR166 in the incipient primordium to delineate the adaxial and abaxial sides. This cascade rests on the adaxial-specific accumulation of miR390, which remains polarized even in molecularly abaxialized $1 b 11$ primordia. The mechanisms that position this small RNA thus act independently of any known polarity determinant and instead may be patterned by positional information inherent to the SAM.

\section{Establishment of organ polarity}

\section{When is the adaxial-abaxial boundary established?}

Lateral organs initiate from the peripheral zone (PZ) at the flank of the SAM, a process governed in part by the distribution of the hormone auxin, as discrete auxin maxima presage the sites of incipient primordia (Reinhardt et al. 2003; Heisler et al. 2005). The PZ is uniquely competent to respond to these maxima, as application of exogenous auxin to the stem cell-containing central zone (CZ) at the meristem tip does not result in organ initiation (Reinhardt et al. 2000, 2003). Expression analyses confirm that many polarity determinants are present in the incipient primordium (e.g., Kerstetter et al. 2001; McConnell et al. 2001; Chitwood et al. 2009). Despite this, the temporal relationship between establishment of the adaxial-abaxial axis and the initiation of lateral organs has not been determined and remains a pressing question in the field.

Initial models of the establishment of organ polarity envisioned a uniform distribution of adaxial and abaxial polarity factors in the incipient leaf (Eshed et al. 2001; Emery et al. 2003; Engstrom et al. 2004). A meristemderived signal, perhaps the hypothetical Sussex signal, would promote adaxial cell fate resulting in polarization along the adaxial-abaxial axis. In the absence of such a signal, the primordium would acquire a default abaxial state. This model is consistent with data from surgical experiments, and the invocation of a meristem-derived signal neatly explains why the adaxial side of leaves always develops closest to the meristem (Fig. 1A,B; Sussex 1951; Reinhardt et al. 2005). However, recent advances in our ability to visualize the dynamics of proteins within the meristem have called this model into question.

Live imaging of Arabidopsis inflorescence meristems reveals that REV is restricted to the adaxial side of the incipient floral meristem, even when it is morphologically indistinguishable from the surrounding inflorescence (Heisler et al. 2005). Similarly, many of the polarity determinants in maize exhibit a polarized expression pattern in the initiating leaf, arguing that adaxial and abaxial factors are not uniformly localized throughout incipient primordia (Juarez et al. 2004a,b; Nogueira et al.
2007). Moreover, molecularly abaxialized $l b l 1$ primordia still exhibit an adaxial-specific accumulation of miR390 (Nogueira et al. 2009). These observations suggest that the incipient organ may be prepatterned into adaxial and abaxial domains. Polarization of the PZ into apical/ centric and basal/outer regions, possibly as a consequence of positional information inherent to the SAM, could form the basis for such a prepattern (Fig. 5). This presents the intriguing possibility that organogenesis occurs at sites where an auxin maximum overlaps a prepatterned adaxial-abaxial boundary, criteria that are met only within the PZ. This model can explain the inability of the $\mathrm{CZ}$ to produce organs, even in the presence of an auxin maximum, and is not without precedent, as organ formation in other taxa occurs at cell fate boundaries as well (see Wolpert 1998). A similar mechanism may also direct leaflet formation in compound leaves. Leaflets form along the margins of leaves, a site with juxtaposed adaxial and abaxial identities, and mutants with perturbed adaxial-abaxial polarity produce fewer leaflets (Kim et al. 2003a,b). Sites of leaflet initiation are also predicted by auxin maxima, implying their formation is similarly driven by the coincidence of these maxima with adaxial-abaxial boundaries (Fig. 5; Barkoulas et al. 2008).

A prepatterned adaxial-abaxial boundary in the PZ may seem incongruent with surgical experiments, suggesting that a mobile meristem-derived signal is required to specify adaxial fate (Sussex 1951; Reinhardt et al. 2005). However, mobile signaling and prepatterning mechanisms are not necessarily mutually exclusive.

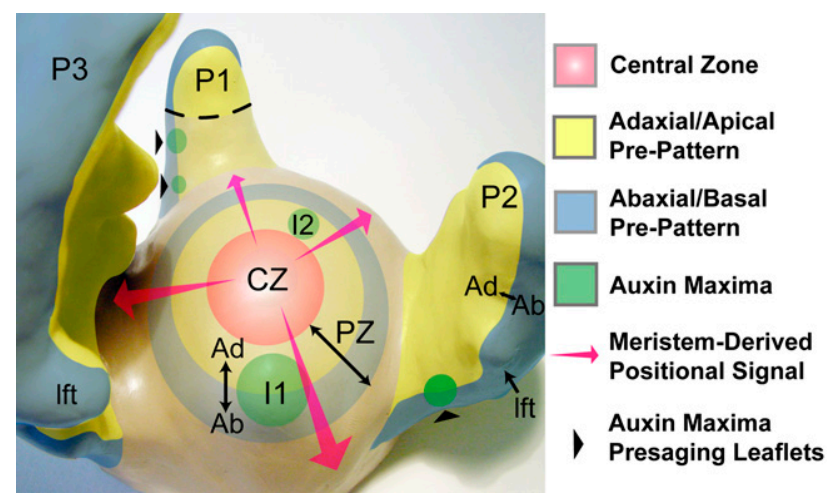

Figure 5. Model for adaxial-abaxial axis specification in the incipient primordium. The SAM comprises a stem cell-containing $\mathrm{CZ}$ and organogenic PZ. The PZ is envisioned to be patterned into apical/centric (gray yellow) and basal/outer (gray blue) regions based on positional information inherent to the SAM, possibly signals derived from the CZ and cells basal to the PZ. A lateral organ initiates at the site where an auxin maximum (green circles) overlaps the boundary between these PZ regions (I1). The same boundary also prepatterns the incipient organ into adaxial and abaxial domains. A meristemderived adaxializing signal (pink arrows) is proposed to maintain this initial polarity until the P2 stage of organ development when maintenance mechanisms within the organ are in place. Compound-leafed species may similarly initiate leaflets (lft) at sites where auxin maxima overlap an adaxial-abaxial boundary at the margins of developing primordia (black arrowheads). 
The acquisition of adaxial-abaxial organ polarity may be divided into two temporal phases: (1) establishment of the adaxial-abaxial axis in incipient primordia in response to a prepatterned $\mathrm{PZ}$, and (2) stable maintenance of this axis in developing organs through antagonistic interactions between polarity determinants and interdomain signaling (Figs. 5, 3A, 4B, respectively). If these maintenance mechanisms need time to resolve, as suggested by surgical experiments (Reinhardt et al. 2005), meristem-derived signals could be required to promote adaxial fate in those primordia that have left the prepatterned PZ, but not yet reached the meristem-independent $\mathrm{P} 2$ stage of development.

\section{Candidates for the proposed upstream positional information}

Assuming prepatterning of the PZ, what positional information could underlie this inherent polarization? One model for adaxial-abaxial polarity suggests that specification of this axis is an extension of embryonic patterning events. The early globular embryo is patterned into a central HD-ZIPIII-expressing region and a peripheral region expressing the KAN genes (McConnell and Barton 1998; Lynn et al. 1999; Kerstetter et al. 2001). The juxtaposition of these two domains drives cotyledon outgrowth; however, it is unclear whether a similar mechanism functions to produce lateral organs in the vegetative SAM. Nonpolar distribution of KAN or HDZIPIII transcripts reduces proximal-distal growth but does not prevent organ outgrowth, and neither does the complete loss of HD-ZIPIII expression in incipient leaf primordia of $1 b 11$ mutants (McConnell et al. 2001; Eshed et al. 2004; Juarez et al. 2004b). In addition, kan1 kan2 kan4 triple mutants develop ectopic leaf-like outgrowths below the cotyledons that correlate with ectopic expression of PINFORMED1 (PIN1), an auxin efflux carrier required to generate auxin maxima (Izhaki and Bowman 2007; Kuhlemeier 2007). This suggests that KAN proteins may define the lower limit of the organogenic zone through inhibition of PIN1 and further implies that KAN should be excluded from the PZ-a supposition supported by the pattern of ZmKAN2 expression in the maize apex (Henderson et al. 2006). Likewise, live imaging shows that while REV expression expands into the adaxial domain of the initiating primordium, it is otherwise restricted to regions more apical to auxin maxima within the PZ (Heisler et al. 2005). The HD-ZIPIII and KAN expression domains thus appear to flank the PZ, rather than mark a prepatterned adaxial-abaxial boundary within it, such that expression of these polarity determinants in incipient primordia is likely a downstream consequence of this prepatterning. This, however, does not exclude the possibility that the HD-ZIPIII-expressing and KAN-expressing domains flanking the PZ contribute positional cues to prepattern this region; this idea would be consistent with the promotive relationship that meristems have with adaxial fate (see above).

In maize, the adaxial accumulation of miR390 is independent of known polarity determinants and, given that its precursor transcripts are expressed more broadly than the mature miRNA, suggests regulators of miRNA biogenesis or stability as candidates to prepattern the PZ (Nogueira et al. 2007, 2009). However, as mutants that perturb tasiR-ARF biogenesis in Arabidopsis do not show polarity defects (Fahlgren et al. 2006; Hunter et al. 2006), regulators of small RNA activity may not contribute to prepatterning the PZ in all plant species.

A signaling molecule often used to create developmental patterns is auxin, although the relationship between auxin and adaxial-abaxial patterning is still unclear. It is intriguing that auxin is transported primarily through the L1 (Reinhardt et al. 2003), particularly in light of surgical experiments demonstrating a role for the L1 in adaxial fate specification (Reinhardt et al. 2005). However, PIN1 polarization toward a centric convergence point within auxin maxima argues against auxin as a candidate for establishing adaxial or abaxial identity in incipient primordia. Instead, auxin may function during the maintenance phase of adaxial-abaxial polarity. After primordia emerge from the SAM, redistribution of PIN1 proteins results in a depletion of auxin primarily from their adaxial side (Heisler et al. 2005; Bayer et al. 2009). This depletion, coupled with the predominantly abaxial localization of its influx carrier AUXIN-RESISTANT1 (Reinhardt et al. 2003), indicates auxin may preferentially accumulate on the abaxial side of developing primordia. This accumulation correlates with the domains of activity of ARF3 and ARF4, two putative targets of auxin (Pekker et al. 2005). Considering that auxin is a mobile signal, it is tempting to speculate that it may act like tasiR-ARFs and contribute positional information that sharpens the adaxialabaxial boundary from the abaxial side (Fig. 4B). However, to formally demonstrate a role for auxin in either the prepatterning of the PZ or the maintenance of adaxialabaxial polarity, more precise knowledge of sites of auxin accumulation will be required (see Vanneste and Friml 2009).

\section{Perspectives}

While the meristem-derived signal first proposed nearly 60 years ago is still unknown, significant advances in our understanding of adaxial and abaxial patterning have been made. Numerous antagonistic interactions between polarity determinants, including members of several transcription factor and small RNA families, create mutually opposing cell fates that form the basis for the separation of adaxial and abaxial domains within the developing organ (Fig. 3A). This rudimentary sorting of cell fates is likely refined and maintained through intercellular communication, perhaps via mobile adaxially derived tasiR-ARFs or an equivalent abaxially derived signal, to achieve the complete and stable separation of these domains throughout organogenesis (Fig. 4B). Identification of such positional signals will be an important advance in the field. Determining how the adaxialabaxial axis is first established also remains a key outstanding question. Recent experiments suggest positional information inherent to the SAM may polarize the PZ to 
prepattern the adaxial-abaxial axis of the incipient primordium. This model predicts that organ formation depends on the coincidence of an auxin maximum with this prepatterned adaxial-abaxial boundary (Fig. 5). Polarity determinants differ in their contribution to adaxialabaxial patterning between species, and the interpretation of a polarized PZ into an adaxial-abaxial axis may similarly vary between plant lineages. Meristem-derived signaling could be required as primordia grow away from this prepatterned PZ until the polarity maintenance network is in place to permanently fix the separation of adaxial and abaxial domains in the developing organ. Identification and characterization of this proposed meristem-derived positional information in diverse plant lineages thus represent an important challenge for the field of leaf polarity.

\section{Acknowledgments}

We thank current and former members of the laboratory for helpful discussions, and Cris Kuhlemeier for valuable comments on the manuscript. We apologize to colleagues whose work was not cited due to space limitations. Research on leaf polarity in the laboratory of M.C.P.T. is supported by grants from the USDA (06-03420) and the NSF (0615752). D.H.C. is an NSF graduate research fellow and a George A. and Marjorie H. Matheson fellow, and Y.P. is the recipient of a fellowship from the Watson School of Biological Sciences supported by Grant GM 065094 from the National Institute of General Medical Sciences, National Institutes of Health.

\section{References}

Addo-Quaye C, Eshoo T, Bartel D, Axtell M. 2008. Endogenous siRNA and miRNA targets identified by sequencing of the Arabidopsis degradome. Curr Biol 18: 758-762.

Adenot X, Elmayan T, Lauressergues D, Boutet S, Bouche N, Gasciolli V, Vaucheret H. 2006. DRB4-dependent TAS3 trans-acting siRNAs control leaf morphology through AGO7. Curr Biol 16: 927-932.

Allen E, Xie Z, Gustafson A, Carrington J. 2005. microRNAdirected phasing during trans-acting siRNA biogenesis in plants. Cell 121: 207-221.

Alvarez J, Pekker I, Goldshmidt A, Blum E, Amsellem Z, Eshed Y. 2006. Endogenous and synthetic microRNAs stimulate simultaneous, efficient, and localized regulation of multiple targets in diverse species. Plant Cell 18: 1134-1151.

Barkoulas M, Hay A, Kougioumoutzi E, Tsiantis M. 2008. A developmental framework for dissected leaf formation in the Arabidopsis relative Cardamine hirsuta. Nat Genet 40: 1136-1141.

Bayer E, Smith R, Mandel T, Nakayama N, Sauer M, Prusinkiewicz P, Kuhlemeier C. 2009. Integration of transport-based models for phyllotaxis and midvein formation. Genes \& Dev 23: 373-384.

Brodersen P, Sakvarelidze-Achard L, Bruun-Rasmussen M, Dunoyer P, Yamamoto Y, Sieburth L, Voinnet O. 2008. Widespread translational inhibition by plant miRNAs and siRNAs. Science 320: 1185-1190.

Byrne M, Barley R, Curtis M, Arroyo J, Dunham M, Hudson A, Martienssen R. 2000. Asymmetric leaves1 mediates leaf patterning and stem cell function in Arabidopsis. Nature 408: $967-971$.

Candela H, Johnston R, Gerhold A, Foster T, Hake S. 2008. The milkweed pod1 gene encodes a KANADI protein that is required for abaxial/adaxial patterning in maize leaves. Plant Cell 20: 2073-2087.

Chapman E, Carrington J. 2007. Specialization and evolution of endogenous small RNA pathways. Nat Rev Genet 8: 884896.

Chitwood D, Nogueira F, Howell M, Montgomery T, Carrington J, Timmermans M. 2009. Pattern formation via small RNA mobility. Genes \& Dev 23: 549-554.

Dai M, Hu Y, Zhao Y, Liu H, Zhou D-X. 2007. A WUSCHELLIKE HOMEOBOX gene represses a YABBY gene expression required for rice leaf development. Plant Physiol 144: 380390.

Emery J, Floyd S, Alvarez J, Eshed Y, Hawker N, Izhaki A, Baum S, Bowman J. 2003. Radial patterning of Arabidopsis shoots by class III HD-ZIP and KANADI genes. Curr Biol 13: 17681774.

Engstrom E, Izhaki A, Bowman J. 2004. Promoter bashing, microRNAs, and KNOX genes. New insights, regulators, and targets-of-regulation in the establishment of lateral organ polarity in Arabidopsis. Plant Physiol 135: 685-694.

Eshed Y, Baum S, Perea J, Bowman J. 2001. Establishment of polarity in lateral organs of plants. Curr Biol 11: 1251-1260.

Eshed Y, Izhaki A, Baum S, Floyd S, Bowman J. 2004. Asymmetric leaf development and blade expansion in Arabidopsis are mediated by KANADI and YABBY activities. Development 131: 2997-3006.

Fahlgren N, Montgomery T, Howell M, Allen E, Dvorak S, Alexander A, Carrington J. 2006. Regulation of AUXIN RESPONSE FACTOR3 by TAS3 ta-siRNA affects developmental timing and patterning in Arabidopsis. Curr Biol 16: 939-944.

Garcia D, Collier S, Byrne M, Martienssen R. 2006. Specification of leaf polarity in Arabidopsis via the trans-acting siRNA pathway. Curr Biol 16: 933-938.

Golz J, Roccaro M, Kuzoff R, Hudson A. 2004. GRAMINIFOLIA promotes growth and polarity of Antirrhinum leaves. Development 131: 3661-3670.

Guilfoyle T, Hagen G. 2007. Auxin response factors. Curr Opin Plant Biol 10: 453-460.

Guo M, Thomas J, Collins G, Timmermans M. 2008. Direct repression of KNOX loci by the ASYMMETRIC LEAVES1 complex of Arabidopsis. Plant Cell 1: 48-58.

Hay A, Tsiantis M. 2006. The genetic basis for differences in leaf form between Arabidopsis thaliana and its wild relative Cardamine hirsuta. Nat Genet 38: 942-947.

Heisler M, Ohno C, Das P, Sieber P, Reddy G, Long J, Meyerowitz E. 2005. Patterns of auxin transport and gene expression during primordium development revealed by live imaging of the Arabidopsis inflorescence meristem. Curr Biol 15: 1899-1911.

Henderson D, Zhang X, Brooks L III, Scanlon M. 2006. RAGGED SEEDLING2 is required for expression of KANADI2 and REVOLUTA homologues in the maize shoot apex. Genesis 44: 372-382.

Huang W, Pi L, Liang W, Xu B, Wang H, Cai R, Huang H. 2006. The proteolytic function of the Arabidopsis 26S proteasome is required for specifying leaf adaxial identity. Plant Cell 18: 2479-2492.

Hunter C, Willmann M, Wu G, Yoshikawa M, dela Luz Guierrez-Nava M, Poethig S. 2006. Trans-acting siRNAmediated repression of ETTIN and ARF4 regulates heteroblasty in Arabidopsis. Development 133: 2973-2981.

Husbands A, Bell E, Shuai B, Smith H, Springer P. 2007. LATERAL ORGAN BOUNDARIES defines a new family of DNA-binding transcription factors and can interact with specific bHLH proteins. Nucleic Acids Res 35: 6663-6671. 
Itoh J-I, Hibara K-I, Sato Y, Nagato Y. 2008. Developmental role and auxin responsiveness of Class III homeodomain leucine zipper gene family members in rice. Plant Physiol 147: 19601975.

Iwakawa $\mathrm{H}$, Iwasaki M, Kojima S, Ueno $\mathrm{Y}$, Soma T, Tanaka $\mathrm{H}$, Semiarti E, Machida Y, Machida C. 2007. Expression of the ASYMMETRIC LEAVES2 gene in the adaxial domain of Arabidopsis leaves represses cell proliferation in this domain and is critical for the development of properly expanded leaves. Plant J 51: 173-184.

Izhaki A, Bowman J. 2007. KANADI and Class III HD-ZIP gene families regulate embryo patterning and modulate auxin flow during embryogenesis in Arabidopsis. Plant Cell 19: 495-508

Juarez M, Kui J, Thomas J, Heller B, Timmermans M. 2004a. microRNA-mediated repression of rolled leaf1 specifies maize leaf polarity. Nature 428: 84-88.

Juarez M, Twigg R, Timmermans M. 2004b. Specification of adaxial cell fate during maize leaf development. Development 131: 4533-4544.

Kerstetter R, Bollman K, Taylor R, Bomblies K, Poethig R. 2001. KANADI regulates organ polarity in Arabidopsis. Nature 411: 706-709.

Kidner C, Martienssen R. 2004. Spatially restricted microRNA directs leaf polarity through ARGONAUTE1. Nature 428: 81-84.

Kim M, McCormick S, Timmermans M, Sinha N. 2003a. The expression domain of PHANTASTICA determines leaflet placement in compound leaves. Nature 424: 438-443.

Kim M, Pham T, Hamidi A, McCormick S, Kuzoff R, Sinha N. 2003b. Reduced leaf complexity in tomato wiry mutants suggests a role for PHAN and KNOX genes in generating compound leaves. Development 130: 4405-4415.

Kim Y-S, Kim S-G, Lee M, Lee I, Park H-Y, Seo PJ, Jung J-H, Kwon E-J, Suh S, Paek K-H, et al. 2008. HD-ZIP III activity is modulated by competitive inhibitors via a feedback loop in Arabidopsis shoot apical meristem development. Plant Cell 20: 920-933.

Kuhlemeier C. 2007. Phyllotaxis. Trends Plant Sci 12: 143-150.

Levine E, McHale P, Levine H. 2007. Small regulatory RNAs may sharpen spatial expression patterns. PLoS Comput Biol 3: e233. doi: 10.1371/journal.pcbi.0030233.

Li H, Xu L, Wang H, Yuan Z, Cao X, Yang Z, Zhang D, Xu Y, Huang H. 2005. The putative RNA-dependent RNA polymerase RDR6 acts synergistically with ASYMMETRIC LEAVES1 and 2 to repress BREVIPEDICELLUS and microRNA165/166 in Arabidopsis leaf development. Plant Cell 17: $2157-2171$.

Lin W-c, Shuai B, Springer P. 2003. The Arabidopsis LATERAL ORGAN BOUNDARIES-domain gene ASYMMETRIC $L E A V E S 2$ functions in the repression of KNOX gene expression and in adaxial-abaxial patterning. Plant Cell 15: 22412252.

Liu Q, Yao X, Pi L, Wang H, Cui X, Huang H. 2009. The ARGONAUTE10 gene modulates shoot apical meristem maintenance and establishment of leaf polarity by repressing miR165/166 in Arabidopsis. Plant J 58: 27-40.

Lynn K, Fernandez A, Aida M, Sedbrook J, Tasaka M, Masson P, Barton M. 1999. The PINHEAD/ZWILLE gene acts pleiotropically in Arabidopsis development and has overlapping functions with the ARGONAUTE1 gene. Development 126: 469-481.

Mallory A, Reinhart B, Jones-Rhoades M, Tang G, Zamore P, Barton M, Bartel D. 2004. MicroRNA control of $P H A B U$ $L O S A$ in leaf development: Importance of pairing to the microRNA 5' region. EMBO J 23: 3356-3364.
Maughan S, Murray J, Bögre L. 2006. A greenprint for growth: Signalling the pattern of proliferation. Curr Opin Plant Biol 9: 490-495.

McConnell J, Barton M. 1998. Leaf polarity and meristem formation in Arabidopsis. Development 125: 2935-2942.

McConnell J, Emery J, Eshed Y, Bao N, Bowman J, Barton M. 2001. Role of PHABULOSA and PHAVOLUTA in determining radial patterning in shoots. Nature 411: 709-713.

McHale N, Koning R. 2004. PHANTASTICA regulates development of the adaxial mesophyll in Nicotiana leaves. Plant Cell 16: 1251-1262.

Montgomery T, Howell M, Cuperus J, Li D, Hansen J, Alexander A, Chapman E, Fahlgren N, Allen E, Carrington J. 2008. Specificity of ARGONAUTE7-miR390 interaction and dual functionality in TAS3 trans-acting siRNA formation. Cell 133: $128-141$.

Navarro C, Efremova N, Golz J, Rubiera R, Kuckenberg M, Castillo R, Tietz O, Saedler H, Schwarz-Sommer Z. 2004. Molecular and genetic interactions between STYLOSA and GRAMINIFOLIA in the control of Antirrhinum vegetative and reproductive development. Development 131: 36493659.

Nogueira F, Madi S, Chitwood D, Juarez M, Timmermans M. 2007. Two small regulatory RNAs establish opposing fates of a developmental axis. Genes \& Dev 21: 750-755.

Nogueira F, Chitwood D, Madi S, Ohtsu K, Schnable P, Scanlon M, Timmermans M. 2009. Regulation of small RNA accumulation in the maize shoot apex. PLoS Genet 5: e1000320. doi: 10.1371/journal.pgen.1000320.

Pekker I, Alvarez J, Eshed Y. 2005. Auxin response factors mediate Arabidopsis organ asymmetry via modulation of KANADI activity. Plant Cell 17: 2899-2910.

Peragine A, Yoshikawa M, Wu G, Albrecht H, Poethig R. 2004. $S G S 3$ and $S G S 2 / S D E 1 / R D R 6$ are required for juvenile development and the production of trans-acting siRNAs in Arabidopsis. Genes \& Dev 18: 2368-2379.

Phelps-Durr T, Thomas J, Vahab P, Timmermans M. 2005. Maize rough sheath2 and its Arabidopsis orthologue ASYMMETRIC LEAVES1 interact with HIRA, a predicted histone chaperone, to maintain knox gene silencing and determinacy during organogenesis. Plant Cell 17: 2886-2898.

Pinon V, Etchells J, Rossignol P, Collier S, Arroyo J, Martienssen R, Byrne ME. 2008. Three PIGGYBACK genes that specifically influence leaf patterning encode ribosomal proteins. Development 135: 1315-1324.

Ponting C, Aravind L. 1999. START: A lipid-binding domain in StAR, HD-ZIP and signalling proteins. Trends Biochem Sci 24: $130-132$.

Reinhardt D, Mandel T, Kuhlemeier C. 2000. Auxin regulates the initiation and radial position of plant lateral organs. Plant Cell 12: 507-518.

Reinhardt D, Pesce E, Stieger P, Mandel T, Baltensperger K, Bennett M, Traas J, Friml J, Kuhlemeier C. 2003. Regulation of phyllotaxis by polar auxin transport. Nature 426: 255-260.

Reinhardt D, Frenz M, Mandel T, Kuhlemeier C. 2005. Microsurgical and laser ablation analysis of leaf positioning and dorsoventral patterning in tomato. Development 132: 15-26.

Sawa S, Watanabe K, Goto K, Liu Y, Shibata D, Kanaya E, Morita E, Okada K. 1999. FILAMENTOUS FLOWER, a meristem and organ identity gene of Arabidopsis, encodes a protein with a zinc finger and HMG-related domains. Genes \& Dev 13: $1079-1088$.

Schrick K, Nguyen D, Karlowski W, Mayer K. 2004. START lipid/sterol-binding domains are amplified in plants and are predominantly associated with homeodomain transcription factors. Genome Biol 5: R41. doi: 10.1186/gb-2004-5-6-r41. 
Siegfried K, Eshed Y, Baum S, Otsuga D, Drews G, Bowman J. 1999. Members of the YABBY gene family specify abaxial cell fate in Arabidopsis. Development 126: 4117-4128.

Sussex I. 1951. Experiments on the cause of dorsiventrality in leaves. Nature 167: 651-652.

Sussex I. 1954. Morphogenesis in Solanum tuberosum L.: Experimental investigation of leaf dorsiventrality and orientation in the juvenile shoot. Phytomorphology 5: 286-300.

Tang G, Reinhart B, Bartel D, Zamore P. 2003. A biochemical framework for RNA silencing in plants. Genes \&. Dev. 17: 49-63.

Timmermans M, Schultes N, Jankovsky J, Nelson T. 1998. Leafbladeless1 is required for dorsoventrality of lateral organs in maize. Development 125: 2813-2823.

Timmermans M, Hudson A, Becraft P, Nelson T. 1999. ROUGH SHEATH2: A Myb protein that represses knox homeobox genes in maize lateral organ primordia. Science 284: 151-153.

Toriba T, Harada K, Takamura A, Nakamura H, Ichikawa $\mathrm{H}$, Suzaki T, Hirano H-Y. 2007. Molecular characterization the YABBY gene family in Oryza sativa and expression analysis of OsYABBY1. Mol Genet Genomics 277: 457-468.

Tsiantis M, Schneeberger R, Golz J, Freeling M, Langdale J. 1999. The maize rough sheath2 gene and leaf development programs in monocot and dicot plants. Science 284: 154-156.

Ueno $\mathrm{Y}$, Ishikawa $\mathrm{T}$, Watanabe $\mathrm{K}$, Terakura $\mathrm{S}$, Iwakawa $\mathrm{H}$, Okada K, Machida C, Machida Y. 2007. Histone deacetylases and ASYMMETRIC LEAVES2 are involved in the establishment of polarity in leaves of Arabidopsis. Plant Cell 19: 445457.

Vanneste S, Friml J. 2009. Auxin: A trigger for change in plant development. Cell 136: 1005-1016.

Vazquez F, Vaucheret H, Rajagopalan R, Lepers C, Gasciolli V, Mallory A, Hilbert J, Bartel D, Crete P. 2004. Endogenous trans-acting siRNAs regulate the accumulation of Arabidopsis mRNAs. Mol Cell 16: 69-79.

Waites R, Hudson A. 1995. phantastica: A gene required for dorsoventrality of leaves in Antirrhinum majus. Development 121: 2143-2154.

Waites R, Selvadurai H, Oliver I, Hudson A. 1998. The PHANTASTICA gene encodes a MYB transcription factor involved in growth and dorsoventrality of lateral organs in Antirrhinum. Cell 93: 779-789.

Wardlaw C. 1949. Experiments on organogenesis in ferns. Growth 13: 93-131.

Wenkel S, Emery J, Hou B-H, Evans M, Barton M. 2007. A feedback regulatory module formed by LITTLE ZIPPER and HD-ZIPIII genes. Plant Cell 19: 3379-3390.

Wolpert L. 1998. Principles of development. Oxford University Press, Oxford.

Wu G, Lin W-c, Huang T, Poethig R, Springer P, Kerstetter R. 2008. KANADI1 regulates adaxial-abaxial polarity in Arabidopsis by directly repressing the transcription of ASYMMETRIC LEAVES2. Proc Natl Acad Sci 105: 16392-16397.

Xu L, Xu Y, Dong A, Sun Y, Pi L, Xu Y, Huang H. 2003. Novel as1 and as2 defects in leaf adaxial-abaxial polarity reveal the requirement for ASYMMETRIC LEAVES1 and 2 and ERECTA functions in specifying leaf adaxial identity. Development 130: 4097-4107.

Xu L, Yang L, Pi L, Liu Q, Ling Q, Wang H, Poethig R, Huang H. 2006. Genetic interaction between the AS1-AS2 and RDR6SGS3-AGO7 pathways for leaf morphogenesis. Plant Cell Physiol 47: 853-863.

Yamaguchi T, Nagasawa N, Kawasaki S, Matsuoka M, Nagato Y, Hirano H-Y. 2004. The YABBY gene DROOPING LEAF regulates carpel specification and midrib development in Oryza sativa. Plant Cell 16: 500-509.
Zhang G-H, Xu Q, Zhu X-D, Qian Q, Xue H-W. 2009. SHALLOT-LIKE1 is a KANADI transcription factor that modulates rice leaf rolling by regulating leaf abaxial cell development. Plant Cell 21: 719-735. 


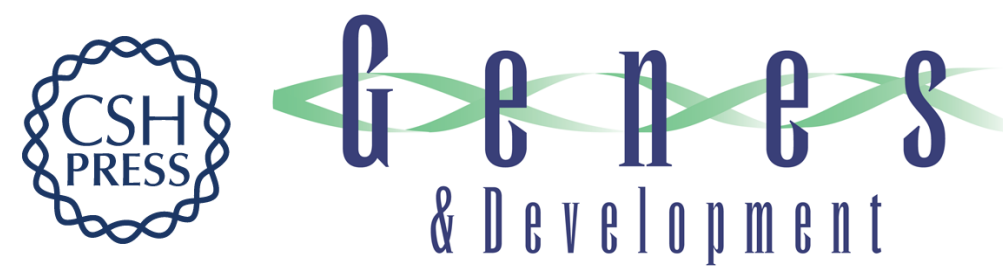

\section{Signals and prepatterns: new insights into organ polarity in plants}

Aman Y. Husbands, Daniel H. Chitwood, Yevgeniy Plavskin, et al.

Genes Dev. 2009, 23:

Access the most recent version at doi:10.1101/gad.1819909

References This article cites 82 articles, 43 of which can be accessed free at: http://genesdev.cshlp.org/content/23/17/1986.full.html\#ref-list-1

License

Email Alerting Receive free email alerts when new articles cite this article - sign up in the box at the top Service right corner of the article or click here.

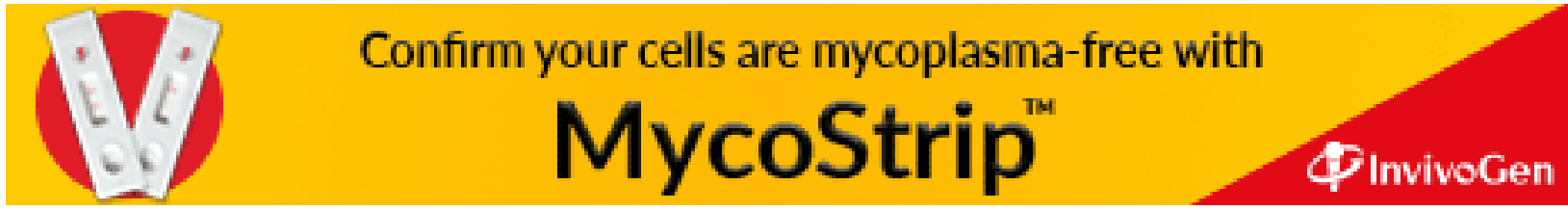

